\title{
Índice de Temperatura y Humedad (THI) respaldado por el cortisol capilar en ganado lechero para la medición de estrés calórico crónico Temperature and Humidity Index (THI) supported by Hair Cortisol in dairy cows for chronic caloric stress measurement
}

\author{
Edna López-Quiñonez ${ }^{1}$ \\ Froylán I. Albarrán-Tamayo 1 (D) 0000-0001-9744-8765 \\ Joel Ramírez-Emiliano ${ }^{2}$ (i) 0000-0001-9813-9120 \\ Bernardo Bañuelos-Hernández ${ }^{1}$ (i) 0000-0003-4399-0887 \\ Andrés Cruz-Hernández ${ }^{3}$ (D) 0000-0005-5507-3878 \\ ${ }^{1}$ Universidad De La Salle Bajío, Campestre, Escuela de Veterinaria \\ $\llbracket$ falbarrant@delasalle.edu.mx \\ ${ }^{2}$ Universidad de Guanajuato, Departamento de Ciencias Médicas \\ ${ }^{3}$ Universidad De La Salle Bajío, Campestre, Escuela de Agronomía \\ (C) Universidad De La Salle Bajío (México)
}

Palabras clave: THI; estrés calórico crónico; cortisol capilar; humedad relativa; velocidad del viento; temperatura ambiental; bienestar animal; medicina veterinaria; ganado lechero; bovinos; producción de leche

Keywords: THI; caloric stress; hair cortisol; relative humidity; wind speed; weather; animal welfare; veterinary Medicine; dairy cattle; bovines; milk production

Recepción: 27 - 01 - 2021 / Aceptación: 09 - 05 - 2021

\section{Resumen}

Introducción: El estrés crónico en el ganado bovino lechero es uno de los mayores problemas para la producción de leche ya que reduce la producción, aumenta las enfermedades en el ganado y ocasiona que se reproduzca menos. Su principal causante es el clima. El estrés calórico ha sido estudiado por muchos años, pero el parámetro más comúnmente usado es el Índice de Temperatura y Humedad (THI), que proviene de las mediciones meteorológicas y no de los cambios que ocurren en el organismo de la vaca.

Método: Para encontrar la relación entre el THI y la respuesta del organismo ante el estrés, muestreamos bimestralmente pelo de 50 vacas Holstein de un establo lechero intensivo automatizado, durante un año; extrajimos el cortisol acumulado y lo medimos mediante la técnica de ELISA. También se obtuvieron las mediciones climatológicas de los reportes meteorológicos locales para calcular los THI y los promedios de cada periodo. Mediante un análisis de regresión múltiple se calculó la correlación de las concentraciones bimestrales de cortisol con los promedios de temperatura, humedad, viento y THI en los mismos periodos. 
Índice de Temperatura y Humedad (THI) respaldado por el cortisol capilar en ganado lechero para la medición de estrés calórico crónico

Resultados: Los cambios en los niveles de cortisol de cada periodo fueron significativamente diferentes al anterior. El análisis de regresión múltiple mostró como principal regresor al THI (p < $0.001)$.

Discusión o Conclusión: Los resultados mostraron que el THI está significativamente relacionado con los niveles de cortisol capilar, esto demuestra que el THI es un parámetro confiable, si no para calcular el estrés fisiológico, sí para saber indirectamente el estrés que puede provocar el clima y tomar decisiones en las explotaciones de ganado lechero.

\begin{abstract}
Introduction: Chronic stress in dairy cattle is one of the biggest problems for milk production as it reduces production, increases disease in cattle and causes them to reproduce less. Its main cause is the weather. Heat stress has been studied for many years, but the most used parameter is the Temperature and Humidity Index (THI), this comes from meteorological measurements and not from the changes that occur in the cow's body.

Method: To find the relationship between THI and the body's response to stress, we sampled hair from 50 Holstein cows every two months from an automated intensive dairy farm, for one year, extracted the accumulated cortisol and measured it using the ELISA technique. We also obtained the climatological measurements from the local meteorological reports to calculate the THI and the averages for each period. By means of a multiple regression analysis, the correlation of the bimonthly cortisol concentrations with the averages of temperature, humidity, wind, and THI in the same periods was calculated.

Results: The changes in cortisol levels in each period were significantly different from the previous one. The multiple regression analysis showed THI as the main regressor $(\mathrm{p}<0.001)$.

Discussion or Conclusion: The results showed the THI significantly related to the levels of cortisol in hair, this shows that the THI is a reliable parameter, if not to calculate the physiological stress, but to indirectly know the stress that weather can cause and make decisions in the farms of dairy cattle.
\end{abstract}

No 27, Vol. 13 (3), 2021. ISSN 2007 - 0705, pp.: 1 - 15

- 2 - 


\section{Introducción}

El calentamiento global se ha convertido en un reto cada vez mayor en distintos ámbitos. Sin embargo, los retos que plantea son considerablemente mayores para los productores de leche (Gauly et al., 2012; Habeeb, Gad y Atta, 2018) ya que la producción láctea alta aumenta la temperatura corporal (Hansen, 2007); si a esto sumamos la temperatura ambiental, el efecto es el estrés calórico (Allen, Hall, Collier y Smith, 2015). Cuando se prolonga, el estrés calórico se vuelve crónico, con el subsecuente incremento de enfermedades y la reducción de la calidad y cantidad de la leche producida (Polsky y von Keyserlingk, 2017), todo esto causado por el incremento en la producción de cortisol (Kadzere, Murphy, Silanikove y Maltz, 2002).

Con la intención de comprender mejor los factores climáticos que afectan más al ganado bovino, durante décadas se ha monitoreado la influencia del frío, el calor y la humedad (Armstrong, 1994; Stull, y otros, 2008; Yano, Shimadzu y Endo, 2014), a partir de estos datos se han desarrollado fórmulas para definir un valor único: el Índice de Temperatura y Humedad (THI) (National Research Counsil, 1971; Bianca, 1962; Thom, 1959; Yousef y Leonard, 1985; Mader, Davis y Brown-Brandl, 2006; West, 1994; Berman, Horovitz, Kaim y Gacitua, 2016). Este índice ha servido como parámetro para estudiar los efectos del clima sobre el ganado lechero. Algunos de esos estudios han comprobado la asociación del THI con la producción de leche en distintas épocas del año (Könyves et al., 2017; Gantner, Mijić, Kuterovac, Solić y Gantner, 2011; Bohmanova, Misztal y Cole, 2007) y diversos puntos geográficos (Bouraoui, Lahmar, Majdoub, Djemali y Belyea, 2002; Zare-Tamami, Hafezian, Rahimi-Mianji, Abdullahpour y Gholizadeh, 2018; OseiAmponsah et al., 2020), así como su relación con los efectos a corto plazo del estrés calórico sobre la fisiología de la vaca (Nascimento et al., 2019).

El estrés a corto plazo o estrés agudo no es un problema para el bienestar del ganado ni para su producción, y sus efectos no pueden considerarse perjudiciales, pues son necesarios para recuperar la homeostasis ante los factores estresantes; por tanto, no deben ser considerados como marcadores del bienestar animal. Por otra parte, si el factor estresante, en este caso el clima, es constante, los efectos fisiológicos mediados por el cortisol se vuelven perjudiciales para su bienestar (Moberg, 2000).

La medición de la cantidad del cortisol como marcador de estrés crónico está limitada por la muestra que se use, ya que se produce de manera constante y es su incremento en la sangre el

que muestra el estrés de los últimos minutos, por lo que puede ser influenciado por el estrés de la

No 27, Vol. 13 (3), 2021. ISSN 2007 - 0705, pp.: 1 - 15 
Índice de Temperatura y Humedad (THI) respaldado por el cortisol capilar en ganado lechero para la medición de estrés calórico crónico

toma de la muestra (Sheriff, Dantzer, Delehanty, Palme y Boonstra, 2011). Por su parte, el cortisol contenido en la saliva muestra el estrés de las últimas horas (Sheriff, Dantzer, Delehanty, Palme, y Boonstra, 2011) y la producción de la saliva puede variar demasiado con el consumo de agua y alimentación (Russell, Koren, Rieder y Van Uum, 2011). Finalmente, para usar el cortisol en el excremento se deberá tener la certeza del sujeto del que proviene cada excreta (Carlitz et al., 2016). En los últimos años, se ha usado la medición del cortisol acumulado en el pelo durante su crecimiento en distintas especies; se obtiene mediante una muestra cuya recolección no es invasiva y contiene el cortisol producido a lo largo de semanas a meses, o incluso años; las fluctuaciones diarias por eventos ocasionales no se reflejan, por lo que es una muestra que representa bien el estrés crónico (Meyer y Novak, 2012).

Diversos estudios realizados en varias especies de mamíferos han comprobado la mayor acumulación de cortisol en el pelo ante estresores como la desnutrición (Bryan, Darimont, Paquet, Wynne-Edwards y Smits, 2013), enfermedades (Trevisan et al., 2017; Park, Kim, Shin y Hwang, 2016) y el clima (Comin et al., 2011). Específicamente en vacas, se ha mostrado un incremento en los niveles de cortisol capilar en la transición de primavera a verano, principalmente en las regiones frías (Uetake et al., 2018).

A pesar de que el THI ha mostrado relación con efectos del estrés a corto plazo, no se ha comprobado su relación con los efectos del estrés a largo plazo, lo que se puede evaluar gracias al cortisol capilar. Por ello el objetivo de este estudio fue comprobar la relación entre el cortisol capilar del ganado bovino lechero y el THI a lo largo de un año.

\section{Método}

\section{Condiciones del estudio}

El estudio contó con la aprobación del Comité de Ética de la Universidad De La Salle Bajío y se realizó en las instalaciones del Centro Agropecuario de Experimentación De La Salle (CADELS), ubicado en La Estancia, en León, Guanajuato México, a una altura de 1, 770 metros sobre el nivel del mar. En el sitio prevalece un clima templado semiseco con primaveras soleadas y baja humedad; en verano se presentan tardes lluviosas, otoños soleados con algunas lluvias e inviernos frescos con vientos fuertes.

En las instalaciones se cría y explota ganado lechero con el mínimo de manejo humano; se usa un sistema automatizado de corrales y un robot ordeñador (VMS V300®DeLaval), brindándole 
el mayor confort a las vacas durante todo su ciclo productivo. A pesar de la automatización, las instalaciones carecen de barreras que eviten el viento, así como de ventiladores o calefactores; por lo anterior, se consideró que es un modelo adecuado para el objetivo de la investigación: con poco estrés por el manejo o alimentación, y sin control del clima.

\section{Sujetos de estudio y muestras}

En febrero de 2016 se seleccionaron 50 vacas de raza Holstein en edad productiva (tabla 1). Se les realizó un corte de pelo al ras de la piel de un área negra a la altura de la escápula; el área del corte fue registrada, y se repitió el corte en la misma zona cada dos meses. Las muestras fueron almacenadas hasta su procesamiento. Cuando se presentaron partos o enfermedades se excluyeron las mediciones del mes para descartar el estrés resultante (tabla 2).

Tabla 1. Características de las vacas en el momento de la inclusión.

Table 1. Cows' characteristics in the time of inclusion.

\begin{tabular}{|c|c|c|c|c|c|}
\hline $\begin{array}{c}\text { Edades } \\
\text { (meses) } \\
\text { Ages } \\
\text { (months) }\end{array}$ & $\begin{array}{c}\text { Partos } \\
\text { previos } \\
\text { Previous } \\
\text { calves }\end{array}$ & $\begin{array}{l}\text { Gestantes } \\
\text { Pregnant }\end{array}$ & $\begin{array}{l}\text { Lactantes } \\
\text { Lactating }\end{array}$ & $\begin{array}{c}\text { Peso }(\mathrm{Kg}) \\
\text { Weight }(\mathrm{Kg})\end{array}$ & $\begin{array}{c}\text { Condición } \\
\text { corporal } \\
(1-5) \\
\text { Body condition } \\
(1-5)\end{array}$ \\
\hline $22 \pm 11.5$ & $0.9 \pm 0.3$ & 2 & 7 & $581.9+61.3$ & $3.25+0.7$ \\
\hline
\end{tabular}

Las edades de inclusión se representan como media y desviación estándar $(\mathrm{n}=50)$. Inclusion ages are presented as mean and standard deviation $(n=50)$.

Tabla 2. Exclusión de mediciones.

Table 2. Measurement exclusión.

\begin{tabular}{|c|c|c|c|c|c|}
\hline \multicolumn{6}{|c|}{2016} \\
\hline $\begin{array}{l}\text { Abril - Mayo } \\
\text { April - May }\end{array}$ & $\begin{array}{l}\text { Junio - Julio } \\
\text { June - July }\end{array}$ & $\begin{array}{l}\text { Agosto - Septiembre } \\
\text { August - September }\end{array}$ & $\begin{array}{l}\text { Octubre - Noviembre } \\
\text { October - November }\end{array}$ & $\begin{array}{l}\text { Diciembre - Enero } \\
\text { December - January }\end{array}$ & $\begin{array}{c}\text { Febrero - Marzo } \\
\text { February - March }\end{array}$ \\
\hline 0 & 1 & 0 & 1 & 12 & 8 \\
\hline
\end{tabular}

Las mediciones excluidas se dieron por partos en todos los casos.

All measurement exclusions were by calving.

\section{Extracción de cortisol}

Adaptando los métodos usados por otros autores (Koren et al., 2002; Accorsi et al., 2008), cada muestra fue lavada dos o tres veces por agitación rigurosa con $2.5 \mathrm{ml}$ de isopropanol, hasta que hubo turbidez (Davenport, Tiefenbacher, Lutz, Novak y Meyer, 2006). Después, la muestra de pelo fue secada con papel absorbente y cortada con tijera en fragmentos de aproximadamente $1 \mathrm{~mm} ; 15$ mg del pelo cortado fueron incubados en $2 \mathrm{ml}$ de metanol durante 24 horas en agitación. 
Posteriormente, el alcohol fue recuperado y evaporado a $50^{\circ} \mathrm{C}$ bajo una corriente de aire. Finalmente, se agregaron 250 ul de buffer salino fosfatado (PBS) y se almacenó a $4^{\circ} \mathrm{C}$ hasta su análisis.

\section{Medición de cortisol}

Se utilizó un kit para ensayo inmuno-absorbente ligado a enzimas (ELISA) de alta sensibilidad (ALPCO ${ }^{\circledR}$ modelo 11-CORHU-E01-SLV; Salem, NH) siguiendo las instrucciones del fabricante. Cada muestra se procesó por duplicado, y la concentración de cortisol de cada una fue calculada usando la curva de calibración del kit. Finalmente, se calculó y ajustó la concentración de cortisol en picogramos por microgramo de la muestra de pelo, ajustando al peso inicial registrado.

\section{Datos meteorológicos}

De los reportes meteorológicos locales (Sistema de Agua Potable y Alcantarillado de León, SAPAL, s.f.), se obtuvieron las mediciones máximas y mínimas de temperatura, humedad relativa y velocidad del viento. Se calcularon los promedios correspondientes a cada periodo, así como los valores del índice de temperatura y humedad correspondientes, de acuerdo con la fórmula del National Research Council (NRC) (National Research Counsil, 1971).

\section{Análisis estadístico}

Se empleó una prueba $T$ de Student para muestras relacionadas para calcular los cambios en las concentraciones de cortisol a lo largo del año y verificar que los cambios fueran significativos. Se realizó un análisis de regresión múltiple paso a paso para identificar al parámetro climático con mayor influencia sobre la concentración de cortisol, incluyendo como variables independientes: la velocidad del viento, la temperatura ambiental promedio, la fluctuación térmica, temperaturas máximas y mínimas, la humedad relativa, precipitación pluvial y el THI. El paquete estadístico fue IBM SPSS Statistics 20th Version.

\section{$\underline{\text { Resultados }}$}

Las condiciones climáticas fueron consistentes con los años anteriores. Con ellas se calculó el THI y se calcularon los promedios de cada bimestre para cada parámetro. Las muestras de pelo 
colectadas fueron procesadas para la extracción y medición del cortisol acumulado en cada bimestre (tabla 3).

Tabla 3. Condiciones climáticas en los periodos evaluados y concentración de cortisol en el pelo.

Table 3. Weather conditions during evaluated periods and hair cortisol concentration.

\begin{tabular}{|c|ccccc|c|}
\hline & \multicolumn{7}{|c|}{2016} & & 2017 \\
\hline $\begin{array}{c}\text { Bimestre } \\
\text { Bimester }\end{array}$ & $\begin{array}{c}\text { Abril - Mayo } \\
\text { April - May }\end{array}$ & $\begin{array}{c}\text { Junio - Julio } \\
\text { June - July }\end{array}$ & $\begin{array}{c}\text { Agosto - Septiembre } \\
\text { August - September }\end{array}$ & $\begin{array}{c}\text { Octubre - Noviembre } \\
\text { October - November }\end{array}$ & $\begin{array}{c}\text { Diciembre - Enero } \\
\text { December - January }\end{array}$ & $\begin{array}{c}\text { Febrero - Marzo } \\
\text { February - March }\end{array}$ \\
\hline Cortisol $(\mathrm{pg} / \mathrm{mg})$ & $43.4 \pm 6.49$ & $34.13 \pm 7.52 *$ & $51.52 \pm 5.52 *$ & $41.14 \pm 5.69 *$ & $49.84 \pm 7.52 *$ \\
\hline $\begin{array}{c}\text { Temperatura } \\
\text { Temperature }\left({ }^{\circ} \mathrm{C}\right)\end{array}$ & $25.02 \pm 1.32$ & $22.70 \pm 0.18$ & $21.59 \pm 0.1$ & $18.82 \pm 0.16$ & $16.56 \pm 0.29$ \\
\hline $\begin{array}{c}\text { Humedad } \\
\text { Humidity }(\%)\end{array}$ & $13.92 \pm 8.32$ & $28.18 \pm 2.24$ & $33.08 \pm 0.46$ & $29.36 \pm 0.45$ & $21.48 \pm 0.53$ \\
\hline THI & $68.40 \pm 1.3$ & $67.20 \pm 0.96$ & $66.40 \pm 0.15$ & $63.10 \pm 2.28$ & $60.50 \pm 1.41$ \\
\hline $\begin{array}{c}\text { Viento } \\
\text { Wind }(\mathrm{km} / \mathrm{h})\end{array}$ & $23.81 \pm 5.16$ & $22.65 \pm 0.99$ & $18.69 \pm 0.15$ & $18.72 \pm 0.38$ & $17.87 \pm 0.38$ \\
\hline
\end{tabular}

Los datos son presentados como media \pm desviación estándar. * Diferencia significativa $(\mathrm{p}<0.05)$

respecto al bimestre anterior, calculados con la prueba $T$ de Student para muestras relacionadas.

Data are presented as media \pm standard deviation. * Significative difference $(\mathrm{p}<0.05)$ versus the previous bimester calculated with Student's T test for related samples.

El análisis de regresión lineal múltiple paso a paso descartó las variables de fluctuación térmica, precipitación pluvial y temperaturas máximas y mínimas. El modelo final es el siguiente:

$$
\mathrm{Y}=\beta 0+\beta 1 \text { Temperatura promedio }+\beta 2 \text { Velocidad del viento }+\beta 3 \text { Humedad relativa }+\beta 4 \mathrm{THI}
$$

Donde: Y es el nivel de cortisol capilar. Este modelo genera un coeficiente de determinación de 0.68 y un error cuadrático medio de 37.73. Los coeficientes de cada regresor se muestran en la tabla 4, en ellos se puede ver que la variable independiente con mayor asociación es el THI.

Tabla 4. Coeficientes del modelo final.

Table 4. Final model coefficients.

\begin{tabular}{|c|c|c|c|c|c|}
\hline \multirow{2}{*}{ Modelo } & \multicolumn{2}{|c|}{ Coeficientes no estandarizados } & Coeficientes tipificados & \multirow{2}{*}{$t$} & \multirow{2}{*}{ Sig. } \\
\cline { 2 - 5 } & $\mathrm{B}$ & Error típico & Beta & -3.605 & 0.000 \\
\hline \begin{tabular}{c} 
(Constante) \\
\hline $\begin{array}{c}\text { Temperatura } \\
\text { Temperature }\end{array}$
\end{tabular} & -482.594 & 133.870 & & -3.571 & 0.000 \\
\hline $\begin{array}{c}\text { Viento } \\
\text { Wind }\end{array}$ & -6.487 & 0.459 & -3.629 & -14.130 & 0.000 \\
\hline $\begin{array}{c}\text { Humedad } \\
\text { Humidity }\end{array}$ & -1.796 & 0.234 & -1.516 & -7.691 & 0.001 \\
\hline THI & 14.417 & 3.175 & 4.667 & 4.541 & 0.000 \\
\hline
\end{tabular}

No 27, Vol. 13 (3), 2021. ISSN 2007 - 0705, pp.: 1 - 15

$$
\text { - } 7 \text { - }
$$


Con la intención de ver más detalles de las interacciones de cada factor climático y su efecto sobre el cortisol, se graficaron las medias de las concentraciones bimestrales de cortisol, y sobre ellas las medias independientes de la temperatura, la humedad y la velocidad del viento (fig. 1).

Agosto - septiembre fue el bimestre con concentraciones más altas de cortisol capilar y de humedad relativa. El segundo periodo con mayor concentración de cortisol fue diciembre - enero, el periodo más frío.

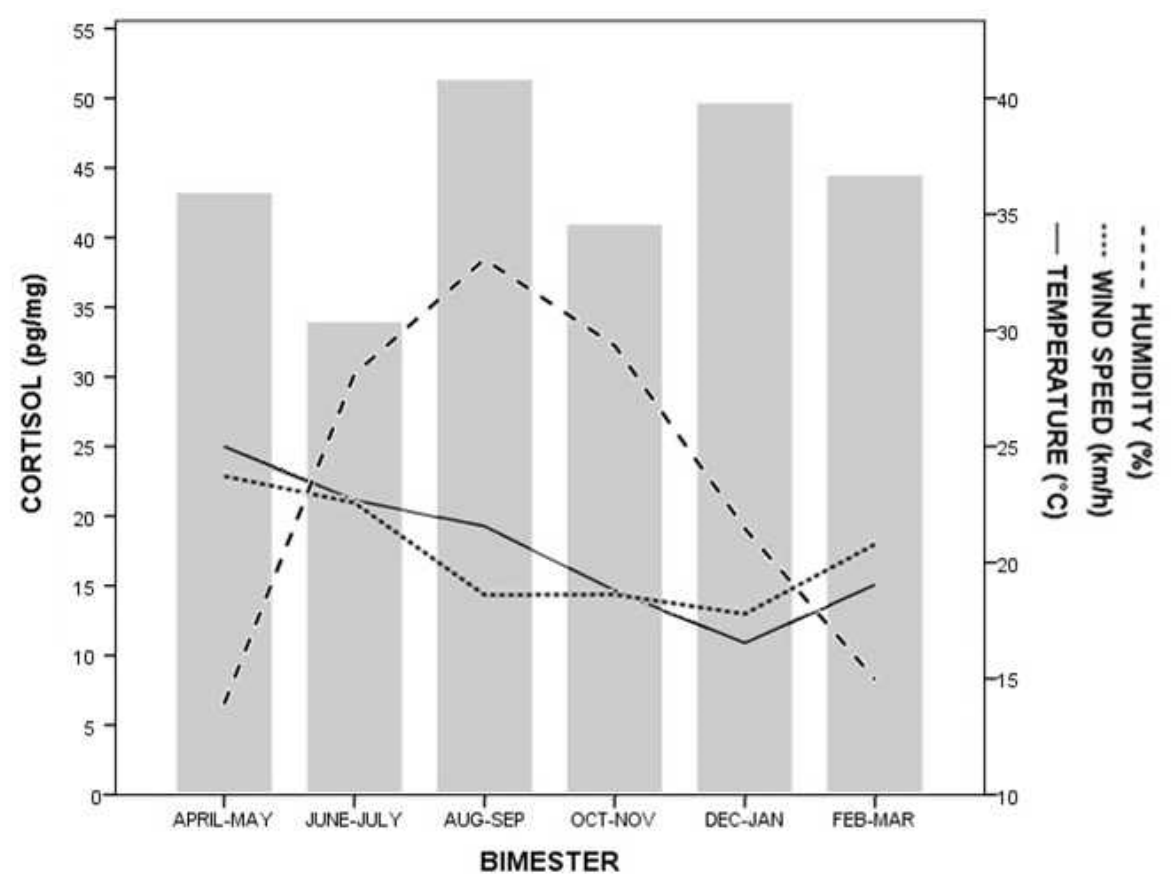

Fig. 1. Las medias de temperatura ambiental, de la velocidad del viento y de la humedad relativa representadas sobre las medias de los niveles de cortisol de las 50 vacas (columnas).

Fig. 1. The means of temperature, wind speed and relative humidity plotted over the means of cortisol levels in the fifty cows (columns).

\section{Discusión y conclusiones}

Es estrés calórico puede ser medido por parámetros fisiológicos como la temperatura corporal o la frecuencia respiratoria (da Costa, Feitosa, Montezuma Jr., de Souza y de Araújo, 2015), sin embargo, estos parámetros son adaptaciones del estrés agudo ante un clima adverso, lo cual no pone en riesgo el bienestar de la vaca (Trevisi y Bertoni, 2009). El estrés crónico necesita ser medido por parámetros que representen periodos mayores, lo cual sí es posible mediante la medición del cortisol acumulado en el pelo. Ya se ha estandarizado su medición para evaluación del estrés crónico por clima incluso descartando variables genéticas (Shi et al., 2021). 
El objetivo de este estudio fue comprobar la relación entre el THI y el estrés crónico, medido por el cortisol capilar. No hay estudios previos que establezcan esta relación, por lo que es importante comprobarla, pues el THI se obtiene de mediciones climáticas, lo cual no refleja el estrés que sufre el organismo de las vacas. Por ello, se eligió como modelo una explotación donde el manejo es poco; se midió el cortisol acumulado en el pelo del ganado cada dos meses durante un año, descartando las mediciones que incluyeran el mes del parto u otros factores estresantes.

En este estudio se han controlado los principales factores que podrían causar estrés crónico y la elevación del cortisol capilar, incluyendo el manejo (Grandin, 1997); el establo donde se realizó el estudio es techado y automatizado desde los corrales hasta la máquina de ordeño, con lo que se minimiza el manejo. También fue controlado el estrés por una nutrición deficiente (Craine, Elmore, Olson y Tolleson, 2010) ya que en esta explotación la nutrición de cada vaca es vigilada, y la ración de alimento es formulada para cada vaca con base en su peso, optimizando así su condición corporal. La salud de los animales, otro factor que, de no cuidarse, genera estrés, está siempre vigilada por médicos veterinarios; para este estudio, cualquier vaca que hubiera enfermado o tenido alguna lesión habría sido excluida. Sin embargo, eso no ocurrió: sólo se excluyeron las mediciones del bimestre en que parió cada vaca, para evitar la posible elevación de los niveles de cortisol en los días previos al parto (Hudson, Mullford, Whittlestone y Payne, 1974), de esta manera el único factor que explica los cambios en los niveles de estrés crónico es el de los cambios climáticos a lo largo del año.

Las concentraciones resultantes de cortisol capilar son mayores a aquellas de otros estudios realizados en vacas. Es importante mencionar que el ganado de esta explotación fue seleccionado para ser altas productoras, las cuales suelen ser más susceptibles al estrés calórico (Coppock, Grant, Portzer, Charles y Escobosa, 1981) lo cual explicaría estos valores de cortisol.

Bucklin et al. (2009) consideran que el THI no es fiel en ganado mantenido bajo sombra, y a pesar de que los valores de THI fueron inferiores al umbral descrito por Brügeman et al. (2012) para causar estrés calórico, se encontraron variaciones significativas a lo largo del año, las cuales permitieron analizar su relación con los cambios del cortisol. Con el análisis de regresión múltiple, además se analizó la relación entre los cambios del cortisol con los parámetros independientes del clima, pero ninguno resultó un mejor regresor que el THI. Se consideró útil estudiar el efecto de cada factor climático sobre el cortisol capilar, para una mejor comprensión del efecto independiente que tiene cada parámetro. 
Al trazar de manera individual la temperatura ambiental, la velocidad del viento y la humedad, se pueden identificar algunas de las influencias sobre el estrés que previamente fueron reportadas por otros autores. Un ejemplo es el periodo con mayor estrés: agosto - septiembre, fue también el periodo con mayor humedad (33\%) y poca velocidad de viento $(18.7 \mathrm{~km} / \mathrm{h})$; ambos factores retrasan la disipación del calor corporal por la piel y respiración (Kibler y Brody, 1950), y a pesar de que la temperatura no es muy alta, sí es superior a la temperatura máxima considerada como confortable por Johnson (1987). El siguiente periodo con más estrés, diciembre-enero, coincide con el clima más frío; aunque la temperatura promedio no bajó del límite del confort, es posible que se presente estrés por hipoxia, ya que las moléculas de oxígeno se dispersan más en el frío (Wood, 1991).

Este parece ser el primer estudio que establece la relación del índice de temperatura y humedad (THI) con el estrés que sufre el organismo. El cortisol capilar es un parámetro directamente relacionado con el estrés crónico, responsable de efectos indeseables para la salud y para la producción del ganado. Este estudio muestra al THI como un parámetro confiable para monitorear el estrés del ganado bovino lechero, a pesar de tratarse de ganado bajo la sombra. Hacen falta estudios que establezcan la misma relación en animales en distintas condiciones y climas.

\section{Agradecimientos}

Agradecemos a la Universidad De La Salle Bajío por el financiamiento y acceso a las instalaciones de su Centro Agropecuario de Investigación. A los médicos veterinarios zootecnistas Aída Arias y Rodrigo Barrios por el acceso al rancho y el aporte de los datos; al médico veterinario zootecnista Leopoldo Ángeles, por su acceso a los laboratorios de la universidad, y al también M.V.Z. Juan Sepúlveda, por su asesoría en la interpretación de los resultados.

\section{$\underline{\text { Referencias }}$}

Accorsi, P., Carloni, E., Valsecchi, P., Viggiani, R., Gamberoni, M., Tamanini, C., y Seren, E. (2008). Cortisol determination in hair and faeces from domestic cats and dogs. Gen Comp Endocrinol, 155(2), 398-402. DOI: https://doi.org/10.1016/j.ygcen.2007.07.002

Allen, J., Hall, L., Collier, R., y Smith, J. (2015). Effect of core body temperature, time of day, and climate conditions on behavioral patterns of lactating dairy cows experiencing mild to 
moderate heat stress. Journal of Dairy Science, 98(1), 118-127. DOI: https://doi.org/10.3168/jds.2013-7704

Armstrong, D. (1994). Heat stress interaction with shade and cooling. Journal of Dairy Science, 77(7), 2044 - 2050. DOI: https://doi.org/10.3168/jds.S0022-0302(94)77149-6

Berman, A., Horovitz, T., Kaim, M., y Gacitua, H. (2016). A comparison of THI indices leads to a sensible heat-based heat stress index for shaded cattle that aligns temperature and humidity stress. International Journal of Biometeorology, 60, 1453-1462. DOI: https://doi.org/10.1007/s00484-016-1136-9

Bianca, W. (1962). Relative importance of dry- and wet-bulb temperatures in causing heat stress in cattle. Nature, 195, 251-252. DOI: https://doi.org/10.1038/195251a0

Bohmanova, J., Misztal, I., y Cole, J. (2007). Temperature-Humidity Indices as Indicators of Milk Production Losses due to Heat Stress. Journal of Dairy Science, 90(4), 1947-1956. DOI: https://doi.org/10.3168/jds.2006-513

Bouraoui, R., Lahmar, M., Majdoub, A., Djemali, M., y Belyea, R. (2002). The relationship of temperature-humidity index with milk production of dairy cows in a Mediterranean climate. Animal Research, EDP Sciences, 51(6), 479-491. DOI: https://doi.org/10.1051/animres:2002036

Brügemann, K., Gernand, E., König von Borstel, U., y König, S. (2012). Defining and evaluating heat stress thresholds in different dairy cow production systems. Archiv Tierzucht, 55(1), 13-24. DOI: https://doi.org/10.5194/AAB-55-13-2012

Bryan, H., Darimont, C., Paquet, P., Wynne-Edwards, K., y Smits, J. (2013). Stress and reproductive hormones in grizzly bears reflect nutritional benefits and social consequences of a salmon foraging niche. PLoS One, 8, 1-10. DOI: https://doi.org/10.1371/journal.pone.0080537

Bucklin, R., Bray, D., Martin, J., Carlos, L., y Carvalho, V. (2009). Enviromental temperatures in Florida dairy housing. Appl. Eng. Agric., 25, 727-735. DOI: https://doi.org/10.13031/2013.28851

Carlitz, E., Miller, R., Kirschbaum, C., Gao, W., Hänni, D., y van Schaik, C. (2016). Measuring hair cortisol concentrations to assess the effect of anthropogenic impacts on wild chimpanzees (Pan troglodytes). PLoS One, 11, e0151870. DOI: https://doi.org/10.1371/journal.pone.0151870 
Comin, A., Prandi, A., Peric, T., Corazzin, M., Dovier, S., y Bovolenta, S. (2011). Hair cortisol levels in dairy cows from winter housing to summer highland grazing. Livestock Science, 138(1-3), 69-73. DOI: https://doi.org/10.1016/j.livsci.2010.12.009

Coppock, C., Grant, P., Portzer, S., Charles, D., y Escobosa, A. (1981). Lactating dairy cow responses to dietary sodium, chloride, and bicarbonate during hot weather. Journal of Dairy Science, 65(4), 566 - 576. DOI: https://doi.org/10.3168/jds.S0022-0302(82)82234-0

Craine, J., Elmore, A., Olson, K., y Tolleson, D. (2010). Climate change and cattle nutritional stress. Global Change Biology, 16(10), 2901-2911. DOI: https://doi.org/10.1111/j.13652486.2009.02060.x

da Costa, A., Feitosa, J., Montezuma Jr., P., de Souza, P., y de Araújo, A. (2015). Rectal temperatures, respiratory rates, production, and reproduction performances of crossbred Girolando cows under heat stress in northeastern Brazil. Int J Biometeorol, 59(11). DOI: 10.1007/s00484-015-0971-4

Davenport, M., Tiefenbacher, F., Lutz, C., Novak, M., y Meyer, J. (2006). Analysis of endogenous cortisol concentrations in the hair of rhesus macaques. Gen Comp Endocrinol, 147, 255-61. DOI: https://doi.org/10.1016/j.ygcen.2006.01.005

Gantner, V., Mijić, P., Kuterovac, K., Solić, V., y Gantner, R. (2011). Temperature-humidity index values and their significance on the daily production of dairy cattle. Mljekarstvo, 61(1), 5663. DOI: https://doi.org/10.1051/animres:2002036

Gauly, M., Bollwein, H., Breves, G., Brügemann, K., Dänicke, S., Daş, G., Demeler, J., Hansen, H., Isselstein, J., Konig, S., Loholter, M., Martinsohn, M., Meyer, U., Potthoff, M., Sanker, C., Schroder, B., Wrage, N., Meibaum, M., von Samson-Himmelstjerna, G., Stinshoff, H. y Wrenzycki, C. (2012). Future consequences and challenges for dairy cow production systems arising from climate change in Central Europe - A review. Animal, 7(5), 1-17. DOI: https://doi.org/10.1017/S1751731112002352

Grandin, T. (1997). Assesment of stress during handling and transport. J Anim Sci, 75(1), 249-257. DOI: https://doi.org/10.2527/1997.751249x

Habeeb, A., Gad, A., y Atta, M. (2018). Temperature-humidity indices as indicators to heat stress of climatic conditions with relation to production and reproduction of farm animals. Int $J$ Biotechnol Recent Adv, 1(1), 35-50. DOI: https://doi.org/10.18689/ijbr-1000107 
Hansen, P. (2007). Exploitation of genetic and physiological determinants of embryonic resistance to elevated temperature to improve embryonic survival in dairy cattle during heat stress. Theriogenology, 68(1), S242-S249. DOI: https://doi.org/10.1016/j.theriogenology.2007.04.008

Hudson, S., Mullford, M., Whittlestone, W., y Payne, E. (1974). Bovine plasma corticoids during partirition. Journal of Dairy Science, 59(4), 744-746. DOI: https://doi.org/10.3168/jds.S0022-0302(76)84267-1

Johnson, H. (1987). Bioclimatology and the adaptation of livestock. New York: Elsevier.

Kadzere, C., Murphy, M., Silanikove, M., y Maltz, E. (2002). Heat stress in lactating dairy cows: A review. Livest. Prod. Sci., 77(1), 59-91. DOI: https://doi.org/10.1016/S0301$\underline{6226(01) 00330-X}$

Kibler, H., y Brody, S. (1950). Environmental physiology with special reference to domestic animals. X. Influence of temperature $5^{\circ}$ to $95^{\circ} \mathrm{F}$ on evaporative cooling from the respiratory and exterior surfaces in Jersey and Holstein cows. University of Missouri. Agricultural Experiment Station. Research bulletin, 461.

Könyves, T., Zlatković, N., Memiši, N., Lukač, D., Puvača, N., Stojšin, M., Halász, A. y Miščević, B. (2017). Relationship of temperature-humidity index with milk production and feed intake of holstein-frisian cows in different year seasons. Thai J Vet Med., 47(1), 15-23.

Koren, L., Mokady, O., Karaskov, T., Klein, J., Koren, G., y Geffen, E. (2002). A novel method using hair for determining hormonal levels in wildlife. Anim Behav, 63(2), 403-6. DOI: https://doi.org/10.1006/anbe.2001.1907

Mader, T., D. M., y Brown-Brandl, T. (2006). Environmental factors influencing heat stress in feedlot cattle. J. Anim. Sci., 84(3), 712-719. DOI: https://doi.org/10.2527/2006.843712x

Meyer, J. y Novak, M. (2012). Minireview: Hair cortisol: a novel biomarker of hypothalamicpituitary- adrenocortical activity. Endocrinology, 153(9), 4120-4127. DOI: https://doi.org/10.1210/en.2012-1226

Moberg, G. (2000). Biological response to stress: implication for animal welfare. En G. Moberg, y G. Mench. The biology of animal stress (123-146). Wallingford, Oxon, UK: CABI Publishing. DOI: https://doi.org/10.1079/9780851993591.0001

Nascimento, F., Aguiar, H., Rodrigues, G., Guimarães, E., Carvalho, E., y Nascimento, M. (2019). What is the best temperature-humidity index equation to indicate heat stress in crossbred 
Índice de Temperatura y Humedad (THI) respaldado por el cortisol capilar en ganado lechero para la medición de estrés calórico crónico

dairy calves in a tropical environment? Ciência Rural, 49(1), e20180132. DOI: http://dx.doi.org/10.1590/0103-8478cr20180132

National Research Counsil. (1971). A Guide to Environmental Research on Animals. Washington, D.C.: National Academy of Science.

Osei-Amponsah, R., Dunshea, F., Leury, B., Cheng, L., Cullen, B., Joy, A., Abhijith, A., Zhang, M. H. y Chauhan, S. (2020). Heat stress Impacts on lactating cows grazing australian summer pastures on an automatic robotic dairy. Animals, 10(5), 869. DOI: https://doi.org/10.3390/ani10050869

Park, S., Kim, S., Shin, N., y Hwang, C. (2016). Elevated cortisol content in dog hair with atopic dermatitis. Jpn. J. Vet. Res., 64(2), 123-129. DOI: https://doi.org/10.14943/jivr.64.2.123

Polsky, L., y von Keyserlingk, M. (2017). Effects of heat stress on dairy cattle welfare. Journal of Dairy Science, 100(11), 8645-8657. DOI: https://doi.org/10.3168/jds.2017-12651

Russell, E., Koren, G., Rieder, M., y Van Uum, S. (2011). Hair cortisol as a biological marker of chronic stress: current status, future directions, and unanswered questions. $\begin{array}{lll}\text { Psychoneuroendocrinology, } & \text { 37(5), 589-601. }\end{array}$ https://doi.org/10.1016/j.psyneuen.2011.09.009

Sheriff, M., D. B., Delehanty, B., Palme, R., y Boonstra, R. (2011). Measuring stress in wildlife: techniques for quantifying glucocorticoids. Oecologia, 166(4), 869-887. DOI: https://doi.org/10.1007/s00442-011-1943-y

Shi, R., Dou, J., Liu, J., Sammad, A., Lou, H., Wang, Y., Guo, G. y Wang, Y. (2021). Genetic parameters of hair cortisol as an indicator of chronic stress under different environments in Holstein cows. Journal of Dairy Science, 104(6), 6985-6999. DOI: 10.3168/jds.201917856

Sistema de Agua Potable y Alcantarillado de León (SAPAL). (s.f.). Recuperado el 20 de Mayo de 2017, de Estaciones Meteorológicas: http://www.sapal.gob.mx/est-meteorologicas

Stull, C., Messam, L., Collar, C., Peterson, N., Castillo, A., Reed, B., Andersen, K. L. y VerBoort, W. (2008). Precipitation and Temperature Effects on Mortality and Lactation Parameters of Dairy Cattle in California. Journal of Dairy Science. 91(12), 4579-4591. DOI: https://doi.org/10.3168/jds.2008-1215

Thom, E. (1959). The discomfort index. Weatherwise, 12(2), 57-59. DOI: https://doi.org/10.1080/00431672.1959.9926960 
Trevisan, C., Montillo, M., Prandi, A., Mkupasi, E., Ngowi, H., y Johansen, M. (2017). Hair cortisol and dehydroepiandrosterone concentrations in naturally Taenia solium infected pigs in Tanzania. Gen. Comp. Endocrinol, 246, 23-28. DOI: https://doi.org/10.1016/j.ygcen.2017.03.007

Trevisi, E., y Bertoni, G. (2009). Some physiological and biochemical methods for acute and chronic stress evaluation in dairy cows. Ital. J. Anim. Sci., 8(1), 265-286. DOI: 10.4081/ijas.2009.s1.265

Uetake, K., Morita, S., Sakagami, N., Yamamoto, K., Hashimura, S., y Tanaka, T. (2018). Hair cortisol levels of lactating dairy cows in cold- and warm-temperate regions in Japan. Anim. Sci. J, 89(2), 494-497. DOI: https://doi.org/10.1111/asj.12934

West, J. (1994). Interactions of energy and bovine somatotropin with heat stress. Journal of Dairy Science, 77(7), 949-956. DOI: https://doi.org/10.3168/jds.S0022-0302(94)77152-6

Wood, S. (1991). Interactions between hypoxia and hypothermia. Annu. Rev. Physiol., 53, 71-85. DOI: https://doi.org/10.1146/annurev.ph.53.030191.000443

Yano, M., S. H., y Endo, T. (2014). Modelling temperature effects on milk production: a study on Holstein cows at a Japanese farm. SpringerPlus, 3, 129. DOI: https://doi.org/10.1186/2193$\underline{1801-3-129}$

Yousef, M., y Leonard, B. (1985). Stress physiology in livestock (Vol. 1). Boca Raton, FL.: CRC Press. DOI: https://doi.org/10.1002/smi.2460020413

Zare-Tamami, F., Hafezian, H., Rahimi-Mianji, G., Abdullahpour, R., y Gholizadeh, M. (2018). Effect of the temperature-humidity index and lactation stage on milk production traits and somatic cell score of dairy cows in Iran. Songklanakarin J. Sci. Technol, 40(2), 379-383. DOI: https://doi.org/10.14456/sjst-psu.2018.36 